\title{
XLVIII. The expression for the electrical conductivity of metals as deduced from the electron theory
}

\section{W.F.G. Swann D.Sc. AR.C.S.}

To cite this article: W.F.G. Swann D.Sc. AR.C.S. (1914) XLVIII. The expression for the electrical conductivity of metals as deduced from the electron theory , Philosophical Magazine Series 6, 27:159, 441-455, DOI: 10.1080/14786440308635108

To link to this article: http://dx.doi.org/10.1080/14786440308635108

曲 Published online: 08 Apr 2009.

Submit your article to this journal $\pi$

Џll Article views: 6

Q View related articles $₫$

47 Citing articles: 4 View citing articles 지 


\section{$\left[\begin{array}{ll}441 & 1\end{array}\right.$}

XLVIII. The Expression for the Electrical Conductivity of Metals as deduced from the Electron Theory. By W. F. G. Swand, D.Sc., A R.C.S., Assistant Lecturer in Physics: at the University of Sheffeld *.

\section{Introduction.}

THE theory of the electrical conductivity of metals has: 1 been worked out on many assumptions. One of the simplest and best known of these methods is that employed by Drude, in which the assumption is made that in the absence of the electric field all the electrons move with the. same velocity, and that the velocity produced by the field in an electron is the velocity which is produced in it while it is travelling between two points of collision, the essential assumption being that at each collision the effect of all previous actions of the field on the electron are wiped out. The value of the conductivity $\sigma$ which has been deduced from these assumptions is

$$
\sigma=\frac{n e^{2} \lambda v}{4 \alpha \theta}+, . . \quad . \quad . \quad .
$$

where $n$ is the number of electrons per c.c., $\lambda$ is the mean free path, $v$ is the velocity, and $\alpha \theta$ is the kinetic energy of a gas molecule at a temperature $\theta$.

The object of Part I. of the present paper is to show that the above assumptions do not lead to (1), but to the formula

$$
\sigma=\frac{n e^{2} \lambda v}{3 \alpha \theta} \text {. . . . . . . . }
$$

The difference between (1) and (2) is partly due to what is, in the opinion of the author, an improper use of the quantity known as the mean free path, and partly due to another cause which will be better understood at a later stage of the paper.

The thermal conductivity $k$ calculated with the proper use of the mean free path gives, for the above case, the ordinarily accepted value $k=\frac{1}{3} n \lambda v \alpha$; and the interesting point is, that while at $0^{\circ} \mathrm{C}$. (1) gives $k / \sigma=6.3 \times 10^{10}$; (2) results in $k / \sigma=4 \cdot 7 \times 10^{10}$. The experimentally found value of $k / \sigma$ for most pure metals is about $6.3 \times 10^{10}$ at $0^{\circ} \mathrm{C}$.; so that the conclusion to be drawn is, that the assumptions on which (1) and (2) are based are nothing like as representative of the

* Communicated by the Author (now of the Carnegie Institution of Washington).

$\dagger$ J. J. 'Thomson, 'Corpuscular Theory of Matter,' p. 56. 
facts, even in the matter of the relation between the thermal and electrical conductivity, as has formerly been supposed. The method adopted by Lorentz, Richardson, and others, in calculating the conductivity are based on quite different principles to that adopted in calculating (1) and (2), the persistence of the velocities playing, at any rate implicitly, a primary part in the development of the theories. The value

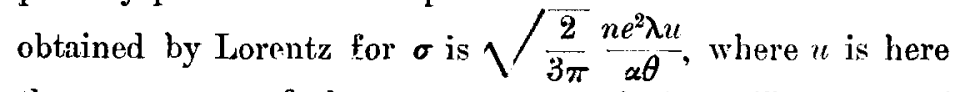
the square root of the mean square velocity. The point of the present paper is not necessarily to suggest that (1) is far wrong in itself, but that the assumptions on which it is based do not lead to it but to (2).

It is perhaps worth while here uttering a word of warning against any feeling which one may have towards assuming that since all methods of calculating the conductivity lead to $\frac{n e^{2} \lambda u}{\alpha \theta}$ multiplied by some numerical factor, therefore the assumptions employed in these methods are all more or less equivalent. If we set out on any hypothesis to calculite $\sigma$ in terms of $n, e, \lambda, u, \alpha \theta$, we are practically bound to find $\frac{u e^{2} \lambda u}{\alpha \theta}$ multiplied by a numerical factor, since this is practically the only way in which these quantities can be combined so as to result in the dimensions of a conductivity, unless we make some most improbable combinations. When we set out to calculate $\sigma$ in terms of $n, e, \lambda, u, \alpha \theta$ the $\frac{n e^{2} \lambda u}{\alpha \theta}$ part is little more than the result of pure algebra, so that it would be obtained if all the physics and all the dynamical principles in our arguments were wrong. The correctness of the numerical factor is in fact the only criterion for the truth of the theory.

It will be convenient first to give the formal proof of (2), and then to put the proof into such a form as to indicate the exact points in which the discrepancy between (1) and (2) has arisen. One or two mathematical details will be relegated to an appendix.

In Part II. of the paper expressions are deduced for the electrical and thermal conductivities on the same assumptions as those on which (2) is deduced, with the exception that the electrons are supposed to move in the absence of the field with the velocities given by Maxwell's law. A method of improving the theoretical value of $k / \sigma$ by making the free path a suitable function of the velocity is also discussed. 
PART I.-Deduction of the expression $\sigma=\frac{n e^{2} \lambda v}{3 \alpha \theta}$.

Let OS (fig. 1) represent the axis of $x$, and let the field $\mathrm{X}$ act from right to left so that the electrons are urged from left to right. We shall proceed to find the flow per square centimetre through the plane perpendicular to the paper and represented by the line MP.

Fig. 1.

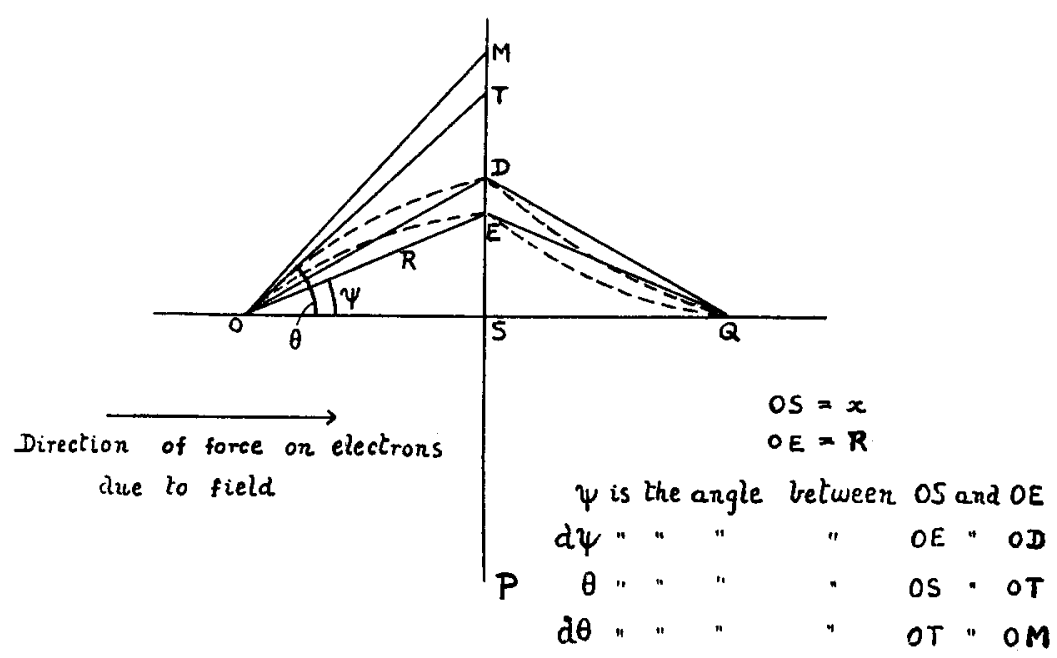

If $\phi$ is measured about $O S$ in the plane perpendicular to the paper, the number $\Delta n$ of electrons starting out from an element $d \omega$ at $O$ having suffered collisions therein, and which are contained within the solid angle $\sin \theta d \theta d \phi$ is

$$
\Delta n=\frac{n v}{4 \pi \lambda} \sin \theta d \theta d \phi d \omega,
$$

where $\theta$ is the angle between OS and the radius vector OT drawn from $0, \lambda$ is the mean free path, and $v$ is the velocity. When the field is off, those of the above electrons which succeed in getting far enough without collisions will strike the plane on the area indicated by MT, but when the field is on they will be bent round. They will travel along paths contained within the dotted lines $\mathrm{OD}$ and $\mathrm{OE}$, and those which get far enough will strike the plane on the area represented by DE.

In the notation of fig. 1 this area amounts to $\frac{\mathrm{R}^{2} \sin \psi}{\cos \psi} d \phi d \psi$. 
The flow per square centimetre through MP due to the group in question is

$$
(\Delta n)_{1}=\frac{n v \sin \theta \cos \psi d \theta}{4 \pi \mathrm{R}^{2} \lambda \sin \psi d \psi} \cdot \epsilon^{-\mathrm{R} / \lambda} d \omega^{*} . \quad .
$$

Now the equations of motion of the electron are

$$
\begin{aligned}
& x=v t \cos \theta+\frac{\mathrm{X} e}{2 m} t^{2}, . \quad \text {. . . . . } \\
& y=v t \sin \theta, \text {. . . . . . . . }
\end{aligned}
$$

where $t$ is the time taken to go from $\mathrm{O}$ to $\mathrm{E}$.

Now

$$
\cos \psi=\frac{x}{\left(x^{2}+y^{2}\right)^{\frac{1}{2}}} \quad \text {. . . . . . }
$$

Substituting from (4) and (5) in (6) and neglecting second order terms $\left(i . e\right.$. terms of the $\left.\underset{\mathrm{X} e}{\mathrm{X}}\left(\frac{\mathrm{X} e x}{m v^{2}}\right)^{2}\right)$, and remembering that in terms multiplied by $\frac{\mathrm{X} e}{m v^{2}}, v t$ may be replaced by $x / \cos \theta$, and that in such terms $\theta$ may be substituted for $\psi$, we readily obtain (see Appendix, problem 1) the result

$$
\cos \psi=\left\{1+\frac{\mathrm{X} e x}{2 m v^{2}} \tan ^{2} \theta\right\} \cos \theta . . .
$$

On differentiating, we readily find

$$
\sin \psi d \psi=\left\{1-\frac{\mathrm{X} e x}{m v^{2}}\left(1+\frac{1}{2} \tan ^{2} \theta\right)\right\} \sin \theta d \theta .
$$

Hence, since $\theta$ may be replaced by $\psi$ in terms multiplying $\mathrm{Xe} / m v^{2}(3)$ becomes

$$
(\Delta n)_{1}=\frac{n v}{4 \pi \mathrm{R}^{2} \lambda}\left\{1+\frac{\mathrm{X} e x}{m v^{2}}\left(1+\frac{1}{2} \tan ^{2} \psi\right)\right\} \cos \psi \cdot \epsilon^{-\mathrm{R} / \lambda} d \omega .
$$

It will now be convenient to take $\mathrm{E}$ as the origin. $\psi$ becomes the angle made by the radius vector to the point $O$ with the normal to MP, and the total number of electrons $\delta n$ reaching $\mathbf{E}$ per square centimetre per second from the ring of volume $2 \pi R^{2} \sin \psi d \psi d R$ passing through $O$ and parallel to the plane $P M$ is

$$
\delta n=\frac{n v}{2 \lambda}\left\{1+\frac{\mathrm{X} e x}{m v^{2}}\left(1+\frac{1}{2} \tan ^{2} \psi\right)\right\} \sin \psi \cos \psi \epsilon^{-\mathrm{R} / \lambda} d \psi d \mathrm{R} .
$$

* In the expression $e^{-\mathrm{R} / \lambda}, \mathrm{R}$ should, strictly speaking, be replaced by the length of the dotted line OE, but it is easy to see that the correction on this account is a second order quantity. 
To obtain the current density across the plane PM due to the electrons coming from the left of the plane, we must multiply $\delta n$ by $e$, and after replacing $x$ by $\mathrm{R} \cos \psi$, integrate first with respect to $R$ from 0 to $\infty$, and then from $\psi=0$ to $\psi=\pi / 2$. Doing this we obtain

$$
\frac{n v e}{2}\left\{\frac{1}{2}+\frac{2 \mathrm{X} e \lambda}{3 m v^{2}}\right\} . \text {. . . . . }
$$

The corresponding quantity due to the flow of electrons from right to left is obtained by replacing $X$ by $-X$ in the above, and the difference of these two current densities represents the resultant current density. Taking this difference and dividing by $\mathrm{X}$ we obtain for the conductivity $\sigma$

$$
\sigma=\frac{2}{3} \frac{n e^{2} \lambda v}{m v^{2}}=\frac{n e^{2} \lambda v}{3 \alpha \theta} *
$$

It will perhaps now be well to indicate the exact reason for the discrepancy between this result and the result indicated by equation (1) and found by Drude. In the deduction of (i) the argument usually given is equivalent to the following: "The electrons may be looked upon as all travelling the distance $\lambda$ between two collisions, the time taken to travel this distance being $\lambda / v$. The velocity created by the field in this time is $\frac{\mathrm{X} e \lambda}{m v}$, and the average velocity created by the field in all the electrons to be found at an instant in any place is $\frac{\mathrm{X} e \lambda}{2 m v}$. The current density is consequently $n e \cdot \frac{\mathrm{X} e \lambda}{2 m v}$, which gives $\sigma=\frac{n e^{2} \lambda v}{4 \alpha \theta}$

The portions in inverted commas must not be looked upon as the arguments of the present author. They simply represent the arguments from which (1) may be considered to have been deduced. It will be noticed that the assumption that the average velocity is half $\frac{\mathrm{X} e \lambda}{m v}$ is equivalent to the assumption that the electrons to be found at any point have on the average travelled a distance $\lambda / 2$, which appears at

* We are not altogether justified in writing $m v^{2}=2 \alpha \theta$, since the existence of a steady temperature involves that all the electrons shall not have the same velocity. The same assumption is, however, involved in (1). It is not this assumption with which I am here particularly concerned, though in Part II, of this paper I have worked out the problem taking account of the velocity distribution.

Phil. Mag. S. 6. Vol. 27. No. 159. March 1914. $2 \mathrm{H}$ 
first sight true on the line of argument that the electrons are on the average in the middle of their journey $\lambda$. The assumption is, however, as we shall see, not true, and is one of the causes of discrepancy between (1) and (2).

Now returning to $(10)$ and omitting the factor $\epsilon^{-\mathrm{R} / \Lambda}$, we have, for the number $(\delta n)_{1}$ of electrons which have suffered collisions in the element specified by $d \mathrm{R}$ and $d \psi$, and which would pass through 1 square centimetre per second at $\mathrm{E}$ if they had suffered no more collisions,

$\langle\delta n)_{1}=\frac{n v}{2 \lambda}\left\{1+\frac{\mathrm{X} e x}{m v^{2}}\left(1+\frac{1}{2} \tan ^{2} \psi\right)\right\} \sin \psi \cos \psi d \psi d \mathrm{R}$.

The number $(\delta n)_{2}$ of the above set to be found in 1 c.c. at $\mathbf{E}$ is obtained by dividing this by $\dot{x}$, the velocity (parallel to the $x$ axis) which the electrons of the above set attain by the time they get to E. In the Appendix, problem (2), it is shown that

$$
\dot{x}=v\left\{1+\frac{\mathrm{X} e x}{m v^{2}}+\frac{\mathrm{X} e x}{2 m v^{2}} \tan ^{2} \psi\right\} \cos \psi,
$$

so that

$$
(\delta n)_{2}=\frac{n}{2 \lambda} \sin \psi \cdot d \mathrm{R} d \psi .
$$

Now the number of the above set which travel a distance between $r$ and $r+d r$ is

$$
\frac{n}{2 \lambda^{2}} \epsilon^{-r / \lambda} d r . d \mathrm{R} \sin \psi d \psi \text {. . . . . }
$$

From the whole range 0 to $\pi / 2$ the number is

$$
\frac{n}{2 \lambda^{2}} \epsilon^{-r / \lambda} d r . d \mathrm{R} \int_{0}^{\pi / 2} \sin \psi d \psi=\frac{n}{2 \lambda^{2}} \epsilon^{-r / \lambda} d r \cdot d \mathrm{R} ;
$$

and taking account of the electrons which have come from the right-hand side of the plane, we have to multiply by 2 , so that we have for the total number $(\delta n)_{3}$ of electrons to be found per c.c. at $\mathbf{E}$ which have come from the shell of thickness $d \mathrm{R}$, and which are in the process of travelling distances between $r$ and $r+d r$,

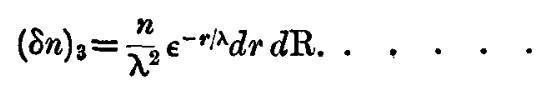

Now keeping our attention on the electrons which travel 
distances between $r$ and $r+d r$, the value of $\Sigma R(\delta n)_{3}$ for this set is

$$
\frac{n}{\lambda^{2}} \epsilon^{-r / \lambda} d r \int_{0}^{r} \mathrm{R} d \mathrm{R}=\frac{n}{\lambda^{2}} \epsilon^{-r / \lambda} \frac{r^{2}}{2} d r ; . . .
$$

and if $l$ is the average distance which all the electrons to be found at $\mathrm{E}$ have travelled

so that

$$
n l=\int_{0}^{\infty} \frac{n}{\lambda^{2}} \epsilon^{-r / \lambda} \frac{r^{2}}{2} d r=n \lambda ; \quad . \quad . \quad .
$$

$$
l=\lambda .
$$

We may notice that if all the electrons had travelled one and only one distance $\lambda$ the right-hand sides of equations (15) and (16) would have both been modified to the same expression, viz. $\frac{n}{\lambda} \cdot \frac{\lambda^{2}}{2}$, so that $l$ would have been equal to $\lambda / 2$, which is the assumption made in the deduction of (1). In fact, though $\lambda$ is the mean free path, we must not say that the average distance which the electrons at $\mathrm{E}$ have travelled is $\lambda / 2$. It is really $\lambda$. If neglect of this consideration were the only error in the usual calculation of (1), the true result would be $\sigma=\frac{n e^{2} \lambda v}{2 \alpha \theta}$; but there is another source of error to which I will now refer.

It is legitimate to argue that since $\lambda$ is the average distance which all the electrons to be found at $\mathrm{E}$ have travelled, the average velocity created by the field is $\frac{\mathrm{X} e \lambda}{m v}$ (which is the argument made in the deduction of (1) after allowance has been made for the fact that the $\lambda / 2$ of that argument should be replaced by $\lambda$ ), but it is not legitimate to say that the resultant current is therefore $\frac{\mathrm{X} e \lambda}{m v} \cdot n e$, the point being that the field has, as it were, sorted out the electrons differently from the two sides of the plane PM. In fact, if each of the electrons at present at $E$ were suddenly robbed of the velocity which the field has given it, there would still be a resultant current there. We can readily see this; for on glancing at fig. 1 we remember that the electrons which have come from $O$ to $E$ have been bent in their path on the way. They originally started out at an inclination $\theta$ to the normal, i. e. at a greater inclination than the angle $\psi$. On the other hand, the electrons which bave come from the element at $Q$

\section{$2 \mathrm{H} 2$}


symmetrically situated on the other side of the plane PM have been bent the other way, they originally started out at a smaller angle to the normal than the angle $\psi$; so that, quite apart from the momentum communicated by the field, there was originally more momentum in the direction from right to left in the set of electrons which are to be found at $\mathbf{E}$ and which have come from the right of PM than there was originally momentum in the direction from left to right in the set of electrons which are to be found at $\mathrm{E}$, and which have come from the left of PM. The two sets of electrons: coming from elements situated symmetrically on the two sides of the plane PM are not corresponding sets, in view of the fact that their initial velocities are in different directions. Thus, it becomes necessary to add to the current density $\frac{n e^{2} \lambda \mathrm{X}}{m v}$ deduced above, the current density due to this cause, which we will now proceed to calculate.

Omitting for the moment the part of the current directly due to the velocity communicated by the field, the electrons specified by (13) constitute a current $\delta i_{1}$ such that

$$
\delta i_{1}=e v(\delta n)_{2} \cos \theta=\frac{n e v}{2 \lambda^{2}} \epsilon^{-r / \lambda} \cos \theta \sin \psi d \mathrm{R} d r ;
$$

so that using (7) and replacing $\theta$ by $\psi$ in the term multiplied by $\frac{\mathrm{X} e x}{m v^{2}}$, we have

$$
\delta i_{1}=\frac{n e v}{2 \lambda^{2}} \epsilon^{-r / \lambda}\left\{1-\frac{\mathrm{X} e x}{2 m v^{2}} \tan ^{2} \psi\right\} \cos \psi \sin \psi d \mathrm{R} d r .
$$

We must now put $x=\mathrm{R} \cos \psi$, then integrate with respect to $\mathrm{R}$ from $\mathrm{R}=0$ to $\mathrm{R}=r$, and then from $r=0$ to $r=\infty$, finally integrating with respect to $\psi$ from $\psi=0$ to $\psi=\frac{\pi}{2}$. The result is

$$
i_{1}=\frac{n e v}{2}\left\{\frac{1}{2}-\frac{\mathrm{X} e \lambda}{3 m v^{2}}\right\} . . . . .
$$

The corresponding quantity due to the flow from the righthand side of PM is obtained by replacing $X$ by $-X$ in (18), and the current resulting from $i_{1}$ and $i_{2}$ is thus

$$
i_{1}-i_{2}=-\frac{n e^{2} \lambda v \mathrm{X}}{3 m v^{2}} \text {. }
$$


The total resultant current density is thus

$$
\frac{n e^{2} \lambda \mathrm{X}}{m v}+i_{1}-i_{2}=\frac{2}{3} \frac{n e^{2} \lambda v \mathrm{X}}{m v^{2}}=\frac{n e^{2} \cdot \lambda v \mathrm{X}}{3 \alpha \theta} *
$$

Giving for the conductivity $\sigma$,

$$
\sigma=\frac{n e^{2} \lambda v}{3 \alpha \theta}
$$

which is the result found on pages $441-445$.

Part II.-Calculation of the electrical conductivity for the case where the velocities of the electrons are distributed according to Marwell's law in the absence of the electrie field, and where the number of electrons starting out in any infinitesimal velocity range, from an element in which they have suffered collisions, is the same when the field is present as when it is absent.

The number of electrons per c.c. having velocities between $c$ and $c+d c$ is, in the absence of the field,

$$
A \epsilon^{-h m c^{2}} c^{2} d c
$$

where

$$
A=\frac{4 n}{\sqrt{\pi}}(h m)^{3 / 2}, \quad \text { and } \quad l m=\frac{3 m}{4 \alpha \theta} .
$$

If in (11) we replace $v$ by $c, n$ by $A \epsilon^{-k m c^{2}} c^{2} d c$, the resulting expression will be the current density $\delta j_{1}$ due to the flow from left to right of that group of electrons which have velocities between $c$ and $c+d c$. The result is

$$
\delta j_{1}=\frac{\mathrm{A}}{2} \epsilon^{-k n c^{2}} c^{2}\left\{\frac{1}{2}+\frac{2 \mathrm{X} e \lambda}{3 m c^{2}}\right\} d c .
$$

The corresponding current density $\delta j_{2}$ due to the flow from right to left is obtained by replacing $X$ by $-X$ in the above, and the resultant current due to all the electrons whose

* It is to be noted that the part $\frac{n e^{2} \lambda \mathrm{X}}{m v}$, i. e. $\frac{n e^{2} \lambda v}{2 \alpha \bar{\theta}}$, represents the only part of the current accounted for by Drude. The fact that he obtained $\frac{n e^{2} \lambda v}{4 \alpha \theta}$ is simply due to the improper use of the quantity $\lambda$ already referred to.

It is interesting to notice that if we were to imagine that all the electrons for some reason or other travelled one and only one distance $\lambda$, we should have $\sigma=\frac{2 n e^{2} \lambda v}{3} \frac{}{\alpha \theta}$. 
volocities lie between $c$ and $c+d c$ is $\delta j_{1}-\delta j_{2}$. Writing $\delta j$ for this we have

$$
\begin{aligned}
\delta j & =\frac{2 \mathrm{AX} e^{2} \lambda}{3 m} \epsilon^{-k m c^{2}} c d c ; \\
\therefore \quad & j=\frac{2 \mathrm{AX} e^{2} \lambda}{3 m} \int_{0}^{\infty} \epsilon^{-k m c^{2}} c d c . . . .
\end{aligned}
$$

On replacing $\mathrm{A}$ and $\mathrm{hm}$ by the values given above and writing $u$ for the square root of the mean square velocity, we obtain

$$
\sigma=\sqrt{\frac{2}{3 \pi}} \cdot \frac{n e^{2} \lambda u}{\alpha \theta}, \ldots . . . .
$$

which happens to be the same result as that obtained by Lorentz by a different method.

\section{The Thermal Conductivity.}

The determination of the thermal conductivity for a gas when the molecules obey Maxwell's law is a well-known problem. We shall, however, briefly survey the steps for the purpose of applying it to the present problem.

If in (10) we put $\mathrm{X}=0$, and replace $n$ by $A \epsilon^{-h m c^{2}} c^{2} d c$, $v$ by $c$, and $\lambda$ by $\lambda_{c}^{*}$ where $\lambda_{c}$ is the free path for velocity $c$, and where the values refer to the plane PM, we shall obtain the number of electrons which reach $E$ per square centimetre per second, which have come from the ring of volume $2 \pi \mathrm{R}^{2} \sin \psi d \psi d \mathrm{R}$, and whose velocities are contained within the limits $c$ and $c+d c$.

We have for the number

$$
\frac{\mathrm{A}}{2 \lambda_{c}} \epsilon^{-h m c^{2}} c^{3} d c \cdot \epsilon^{-\mathbf{R} / \lambda_{c}} d \mathbf{R} \sin \psi \cos \psi d \psi
$$

Remembering that $\alpha \theta$ is the mean kinetic energy of an electron at a temperature $\theta$, we have for the transference of energy $Q_{1}$ per square centimetre per second across the plane $P M$ at $E$, the result

$\mathrm{Q}_{1}=\frac{\mathrm{A}}{2} \int_{0}^{\infty} \frac{\epsilon^{-h m c^{2}}}{\lambda_{c}} c^{3} d c \int_{0}^{\pi / 2} \sin \psi \cos \psi d \psi \int_{0}^{\infty} \epsilon^{-\mathrm{R} / \lambda_{c}}\left\{\alpha \theta-\alpha \frac{d \theta}{d x} \cdot \mathrm{R} \cos \psi\right\} d \mathrm{R}$.

The corresponding quantity representing the flow from right to left is obtained by replacing $\frac{d \theta}{d x}$ by $\frac{-d \theta}{d x}$, and the resultant thermal current density is obtained by subtracting 
these two quantities. The conductivity $k$ is obtained by dividing this by $\frac{d \theta}{d x}$. We thus have

$$
k=\frac{A \alpha}{3} \int_{0}^{\infty} \epsilon^{-h m} c^{2} c^{3} \lambda_{c} d c=\frac{4 n \alpha}{3 \sqrt{ } \pi}(h m)^{\frac{3}{2}} \int_{0}^{\infty} \epsilon^{-h m c^{2}} c^{3} \lambda_{o} d c .
$$
find

For the present case, where we take $\lambda_{c}$ as constant, we

$$
k=\frac{2}{3} \sqrt{\frac{2}{3 \pi}} n \lambda u \alpha,
$$

and using (20)

$$
\frac{k}{\sigma}=\frac{2 \alpha^{2} \theta}{3 e^{2}}=3.6 \times 10^{10} \text { at } 0^{\circ} \mathrm{C} .,
$$

which is very far from the experimental value.

If the mean free path were a function of the velocity, as might conceivably happen if the centres inside the atoms with which the collisions occur were in motion, the expressions for $k$ and $\sigma$ would require modification. $\lambda_{c}$ would have to be retained inside the integral in equation (21), and if $\lambda_{c}$ were a function only of the initial velocity with which the electron started out after collision, the only modification which would be necessary in the calculation of $\sigma$ would be to retain $\lambda_{c}$ inside the integral in (19), so that we should have

$$
\frac{k}{\sigma}=\frac{\alpha m}{2 e^{2}} \frac{\int_{0}^{\infty} \epsilon^{-h m c} c^{2} c^{3} \lambda_{c} d c}{\int_{0}^{\infty} \epsilon^{-h m c^{2}} c \lambda_{c} d c} .
$$

If, for example, $\lambda_{c}$ were proportional to $c^{n}$, we should have

$$
\frac{k}{\sigma}=\frac{\alpha}{2 e^{2} h} \cdot \frac{\Gamma\left(\frac{n}{2}+2\right)}{\Gamma\left(\frac{n}{2}+1\right)}=\frac{2 \alpha^{2} \theta}{3 e^{2}}\left(\frac{n}{2}+1\right),
$$

so that if $\lambda c$ were proportional to $c^{\frac{5}{2}}$, we should have

$$
\frac{k}{\sigma}=6 \cdot 3 \times 10^{10},
$$

which is just the correct value.

Unfortunately, when we form a physical picture of the phenomenon it does not seem reasonable to try in the problem 
of electrical conduction to take full cognizance of the essential phenomena by expressing the mean free path as a function of the velocity with which the electron started its journey, since we should expect that the factor which would determine whether an electron suffered a collision in any given element would be its velocity while in that element, and this would depend upon the distance which the electron had travelled resolved parallel to the field as well as upon its initial velocity. In fact, it is probably more reasonable to state the case in the following way.

Let $\lambda_{c}$ be the mean free path for a velocity $c$ in the absence of the field, and let the fraction of $\mathrm{N}$ electrons which suffer collisions in passing through an element of thickness $d \zeta$ be $f(\dot{\zeta}) d \zeta$, so that

$$
\frac{-d N}{d \zeta}=\mathrm{N} f(\dot{\zeta}) \text { and } \mathrm{N}=\mathrm{N}_{0} \epsilon^{-\int_{0}^{\zeta} f(\dot{\zeta}) d \zeta}
$$

When $\dot{\zeta}$ is a constant over the path of an electron and equal to the velocity $c$ with which the electron started out, the integral becomes $\zeta f(c)$, which shows that $f(c)=1 / \lambda_{c}$. In order to obtain the number of electrons $\delta n^{\prime}$ coming out from the ring of volume $2 \pi \mathrm{R}^{2} \sin \psi d \psi d \mathrm{R}$ (p. 444) and passing through $\mathrm{E}$ per square centimetre per second, and whose velocities lie between $c$ and $c+d c$, it is necessary to replace $\frac{n v}{2 \lambda} \mathrm{n}$ equation (10) by $\frac{A \epsilon^{-h m c^{2} c^{2} c d c}}{2 \lambda_{c}}, v$ by $c$, and $\epsilon^{-\mathrm{R} / \lambda}$ by $\epsilon^{-\int_{0}^{R} f\left(c^{2+}+\frac{2 X_{e x}}{m}\right)^{\frac{1}{2}} d \mathrm{R}}$, since if $c$ is the velocity with which an electron starts out, its velocity after travelling a distance whose component parallel to the field is $x$ amounts to $\left(c^{2}+\frac{2 \mathrm{X} e x}{m}\right)^{\frac{1}{2}}$.

Now if we expand $f\left(c^{2}+\frac{2 \mathrm{X} e x}{m}\right)^{\frac{1}{2}}$ by Taylor's theorem,

then integrate with respect to $R$ remembering that $x=R \cos \psi$, and finally replace $f(c)$ by $1 / \lambda_{e}$, we readily find

$\epsilon^{-\int_{0}^{\mathrm{R}} f\left(c^{2}+\frac{2 \mathrm{X} e x}{m}\right)^{\frac{2}{2}} d \mathfrak{R}}=\epsilon^{-\mathrm{R} / \lambda_{c}} \frac{\mathbf{R}^{2} \mathrm{\epsilon} e \cos \psi}{2 m \lambda_{c}^{2} c} \cdot \frac{\partial \lambda_{c}}{\partial c}=\left\{1+\frac{\mathbf{R}^{2} \mathrm{X} e \cos \psi}{2 m \lambda_{c}^{2} c} \cdot \frac{\partial \lambda_{c}}{\partial c}\right\} \epsilon^{-\mathbf{R} / \lambda_{c}}$.

* It will be noticed that the total number of electrons starting out from an element after suffering collisions therein cannot be alfected by the field to the first power of $\frac{\mathrm{Xe}}{m c^{2}}$. 
Thus

$\delta n^{\prime}=\frac{A \epsilon^{-h m c^{2}}}{2 \lambda_{c}} c^{3} d c\left\{1+\frac{\mathrm{X} e x}{m c^{2}}\left(1+\frac{1}{2} \tan ^{2} \psi+\frac{\mathrm{R} c}{2 \lambda_{c}^{2}} \cdot \frac{\partial \lambda_{c}}{\partial c}\right)\right\} \epsilon^{-\mathrm{R} / \lambda_{c}} \sin \psi \cos \psi d \psi d \mathrm{R}$

The remainder of the work is now exactly analogous to that carried out on pages 445 and 449 , and we obtain

$$
\sigma=\frac{2}{3} \frac{A e^{2}}{m i}\left\{\int_{0}^{\infty} \epsilon^{-h m c^{2}} \lambda_{c^{c}} c d c+\frac{1}{2} \int_{0}^{\infty} \epsilon^{-h m e^{2}} \frac{\partial \lambda_{c}}{\partial c} \cdot c^{2} d c\right\} .
$$

Integrating the second term by parts we finally obtain

$$
\sigma=\frac{2}{3} \mathrm{~A} e^{2} h \int_{0}^{\infty} \epsilon^{-h m c^{2}} \lambda c^{3} d c
$$

Thus, using (21) we have

$$
\frac{k}{\sigma}=\frac{2}{3} \frac{\alpha^{2} \theta}{e^{2}}
$$

The result is thus independent of the manner in which $\lambda_{c}$ varies with the velocity.

\section{Appendix.}

Problem (1).

To show that $\cos \psi=\left\{1+\frac{\mathrm{X} e x}{2 m v^{2}} \tan ^{2} \theta\right\} \cos \theta$.

Subsituting in (6) from (4) and (5) we have, neglecting second-order quantities,

$$
\begin{aligned}
\cos \psi & =\frac{\left(v \cos \theta+\frac{\mathrm{X} e}{2 m} t\right) t}{\left\{v^{2} t^{2} \cos ^{2} \theta+\frac{\mathrm{X} e v}{m} t^{3} \cos \theta+v^{2} t^{2} \sin ^{2} \theta\right\}^{\frac{3}{2}}} \\
& =\left(1+\frac{\mathrm{X} e t}{2 m v \cos \theta}\right)\left(1+\frac{\mathrm{X} e t \cos \theta}{m v}\right)^{-\frac{1}{2}} \cos \theta .
\end{aligned}
$$

Putting $v t \cos \theta=x$, we readily obtain

$$
\cos \psi=\left\{1+\frac{\mathrm{X} e x}{2 m v^{2}} \tan ^{2} \theta\right\} \cos \theta
$$


454 The Eapression for Electrical Conductivity of Metals.

Problem (2).

To show that $\dot{x}=v\left\{1+\frac{\mathrm{X} e x}{m v^{2}}+\frac{\mathrm{X} e x}{2 m v^{2}} \tan ^{2} \psi\right\} \cos \psi$.

From (4) we have

$$
\dot{x}=v \cos \theta+\frac{\mathrm{X} e}{m} t=\left\{1+\frac{\mathrm{X} e x}{m v^{2} \cos ^{2} \theta}\right\} v \cos \theta .
$$

Replacing $\cos \theta$ by its value in terms of $\cos \psi$ as found from Problem (1), we have

$$
\begin{aligned}
\dot{x} & =\left\{1+\frac{\mathrm{X} e x}{m v^{2} \cos ^{2} \theta}-\frac{\mathrm{X} e x}{2 m v^{2}} \tan ^{2} \theta\right\} v \cos \psi \\
& =\left\{1+\frac{\mathrm{X} e x}{m v^{2}}+\frac{\mathrm{X} e x}{2 m v^{2}} \tan ^{2} \psi\right\} v \cos \psi .
\end{aligned}
$$

The University of Sheftield.

July 30th, 1913.

\section{Note added Dec. 26th, 1913.}

Since the above paper was written, certain further matters in relation to the history of expressions (1) and (2) have come to my notice.

Before Drude published the expression (1), and as early as 1898, E. Riecke (Wied. Ann. Bd. lxvi. pp. 353 et seq., 1898), in a paper in which both positive and negative carriers were included, obtained an expression which when interpreted in terms of the modern. electron theory would amount to $\sigma=\frac{n e^{2} \lambda v}{2 \alpha \theta}$. In the same year, and still before the publication of Drude's paper, Riecke found it necessary to introduce a correction into his calculation which actually results in the expression $\sigma=\frac{n e^{2} \lambda v}{3 \alpha \theta}$ deduced above (see Wied. Ann. Bd. lxvi. p. 1199, 1898). In spite of this fact, Drude's result $\sigma=\frac{n e^{2} \lambda v}{4 \alpha \theta}$ is still quoted in all the well known books dealing with the subject; and this fact is the more remarkable since the difference between the two formula is not the outcome of any difference in the fundamental assumptions in the two methods, but is simply due to an error in Drude's work, which, as is shown in the present. paper, is of a purely mathematical nature. 
While this paper was in the press, I learned from Dr. Niels Bohr, during conversation, that he has referred to. the discrepancy between the two expressions in a footnote on page 54 of his 'Dissertation' (which is printed in Danish), and has remarked that it is due to the erroneous method by which Drude calculated the mean velocity communicated to the electrons by the field. In spite of these facts, the parts. of the present paper dealing with this matter will I think be found of interest in indicating more precisely the exact point, or rather points (for there are two) which are involved in the rather subtle explanation of the discrepancy.

\section{On Nuclear Electroms. By A. VAN DEN BRoEK *}

THAT the intra-atomic charge is equal not to half the 1 atomic weight, but to the number $M$ each element occupies in Mendelejeff's series arranged in order of increasing atomic weight, follows from the ratio of the scattering of a-particles per atom divided by the square of that number being a real constant $(18.7 \pm 0.3$ for $\mathrm{Cu}, \mathrm{Ag}$, $\mathrm{Sn}$, $\mathrm{Pt}, \mathrm{Au}$ ), as according to Rutherford must be the case for that ratio divided by the square of the charge, while if the square of half the atomic weight is taken, these numbers show systematic diminution from, if as mean value 18.7 too is taken, $20 \cdot 6$ for $\mathrm{Cu}$ to 17.5 for $\mathrm{Au} \dagger$.

TABLE I.

\begin{tabular}{|c|c|c|c|c|c|c|}
\hline & Cu. & Ag. & Sn. & Pt. & Au. & Mean. \\
\hline Seattering $\div \frac{A^{2}}{5 \cdot 4}$ & $20 \cdot 6$ & $18 \cdot 9$ & $18 \cdot 1$ & $17 \cdot 8$ & 17.5 & $18 \cdot 6$ \\
\hline $\mathbf{M}$ & 29 & 47 & 50 & 82 & 83 & \\
\hline Scattering $\div \mathbf{M}^{2}, \ldots$ & $18 \cdot 5$ & $18 \cdot 4$ & $19 \cdot 0$ & $18 \cdot 6$ & $18 \cdot 4$ & $18 \cdot 6$ \\
\hline
\end{tabular}

As these nuclear charges for $\mathrm{U}$ and $\mathrm{Pb}$, as for $\mathrm{Th}$ and $\mathrm{Bi}$, give other differences than the number of positive charges lost by expulsion of the $\alpha$-particles (the nuclear charge difference being 10 for $\mathrm{U}$ and $\mathrm{Pb}$, and 7 for $\mathrm{Th}$ and $\mathrm{Bi}$,

* Communicated by the Author.

$\dagger$ Geiger \& Marsden, Phil. Mag. xxv. p. 604 (1913). 